\title{
Perihilar Cholangiocarcinoma on Biliary Anatomical Variant. Case Report
}

\author{
Florin Botea ${ }^{1}$, Diana Nicolaescu ${ }^{1}$, Alexandru Barcu ${ }^{1}$, Nausica $\mathrm{Picu}^{1}$, Radu Dumitru ${ }^{2}$, Ruxandra Fota ${ }^{3}$, \\ Vlad Herlea ${ }^{4}$, Irinel Popescu ${ }^{1}$
}

Corresponding author: Irinel Popescu, MD

"Dan Setlacec" Center of General Surgery and Liver Transplantation Fundeni Clinical Institute, Fundeni Street no 258, Bucharest, Romania E-mail: irinel.popescu220@gmail.com
1"Dan Setlacec" Center of General Surgery and Liver Transplantation, Fundeni Clinical Institute, Bucharest, Romania ${ }^{2}$ Department of Radiology, Fundeni Clinical Institute, Bucharest, Romania ${ }^{3}$ Intensive Care Unit, Fundeni Clinical Institute, Bucharest, Romania ${ }^{4}$ Department of Pathology, Fundeni Clinical Institute, Bucharest, Romania

\section{ABSTRACT}

Background. Perihilar cholangiocarcinoma (PCC), defined as tumors involving or in close vicinity to the main bile duct confluence, is the most frequent type of cholangiocarcinoma (CC). Nevertheless, PCC occurring on biliary anatomical variant is a rare condition, difficult to diagnose and to establish the proper surgical approach.

Case presentation. A 59-year old male, with normal liver function, is incidentally diagnosed with PCC of the left hepatic duct (LHD), associated with a biliary anatomical variant consisting in right posterior hepatic duct (RPHD) entering the LHD. The patient underwent left hemihepatectomy with en-bloc resection of segment 1 and hilar lymph node dissection, and cholangiojejunostomy for the RPHD stump.

Results. The intraoperative and postoperative outcome were remarkably uneventful. Negative resection margins of the operative specimen were observed at pathological examination. No local recurrence occurred during a 2-yr follow-up.

Conclusion. Careful preoperative assessment and identification of anatomical variants are often the key to a successful surgical treatment in PCC.

Key words: perihilar cholangiocarcinoma, bile duct cancer, biliary anatomical variant, hemihepatectomy, en-bloc resection of segment 1.

\section{INTRODUCTION}

Cholangiocarcinoma (CC) represents about $10 \%$ of primary malignant liver tumors, and about $2 \%$ of all malignancies (1), with an incidence that is continuously increasing in the past decades (2). CC originates from the malignant transformation of the bile duct epithelium along the biliary tree, encompassing three main subgroups of tumors with specific epidemiological, clinical, therapeutic, and prognostic features (3):

- intrahepatic cholangiocarcinoma (ICC), representing less than $10 \%$, 
originating from small intrahepatic bile ducts;

- perihilar cholangiocarcinoma (PCC) (Klatskin tumor), representing the majority of cases (about $65 \%)$, defined as tumors involving or in close vicinity to the main bile duct confluence;

- distal cholangiocarcinoma (DCC), representing about 25\%.

PHC in close vicinity but not involving the biliary confluence is very common in case of the common hepatic duct, but very rarely encountered in left or right hepatic duct. This is most probably due to the fact that $\mathrm{PHC}$ originating from left or right hepatic duct is commonly diagnosed in late stages, when arrives to invade the main biliary confluence. Surgical management is the best option for cure in PCC (4). Surgically relevant anatomical variants of these bile ducts are as frequent as $25-37 \%(5,6,7)$.

PCC arising on biliary ducts with anatomical variants complicate even more the clinical and paraclinical presentation, and such patients are often erroneously considered unresectable, due to the fact that anatomical variants are practically never considered in this clinical settings.
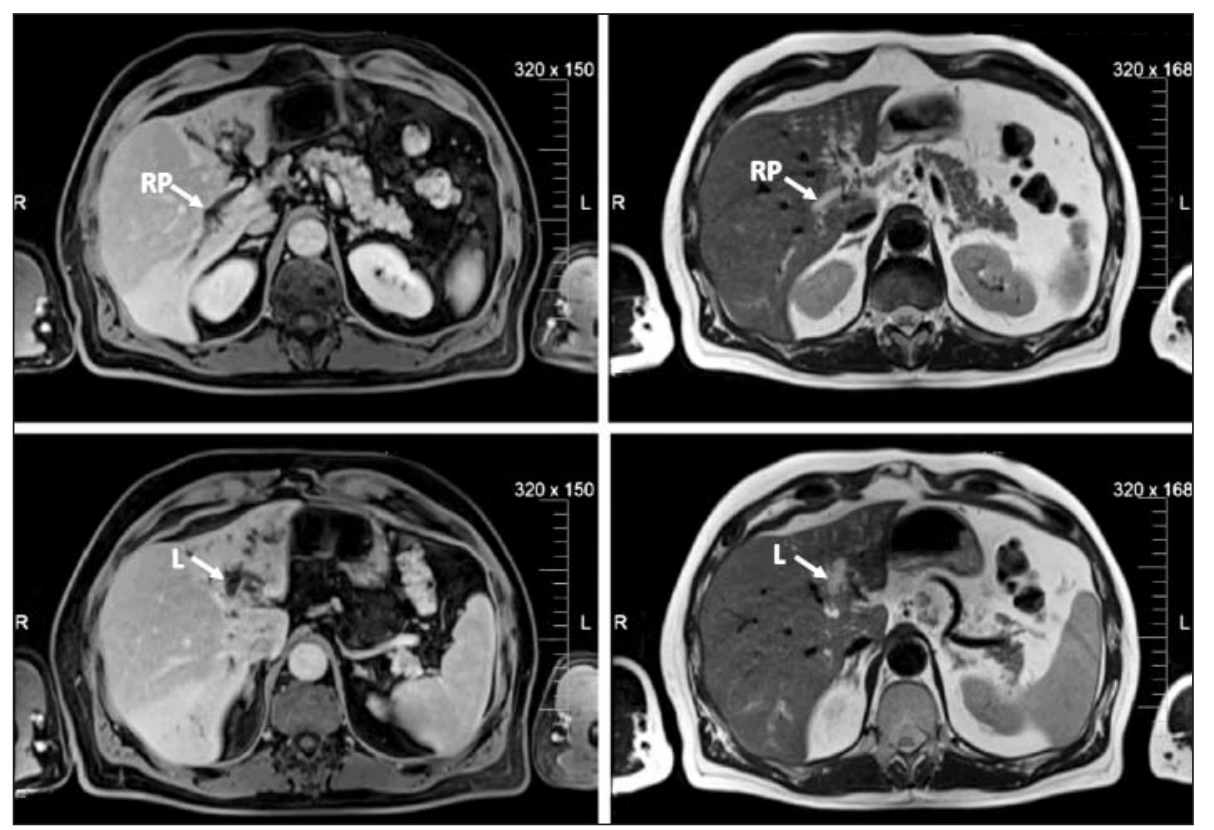

Figure 1 - Contrast-enhanced magnetic resonance cholangiography detected a PCC of the left hepatic duct (L) with right posterior hepatic duct (RP) draining into it. Note the intrahepatic bile duct dilatation of the left hemiliver and the right posterior section, but not of the right anterior section, with a non-dilated right anterior hepatic duct (RA) draining directly into the main bile duct.

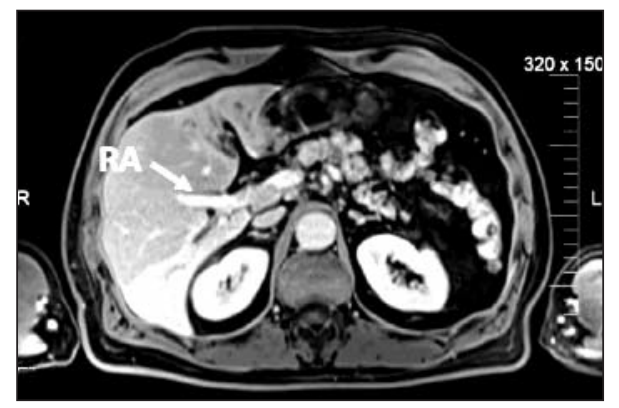

The present paper presents such a case, with a left duct PCC arising on a particular anatomical variant of hepatic biliary ducts.

\section{CASE REPORT}

A 52-year old male, heavy smoker, with a history of lower limbs obstructive arteriopathy, without jaundice, is incidentally diagnosed with intrahepatic bile duct dilatation during an contrast-enhanced computed tomography angiography for arterial assessment. A contrast-enhanced magnetic resonance cholangiography detected a PCC with intrahepatic bile duct dilatation in the left hemiliver and the right posterior section (segments 6 and 7 according Couinaud classification) (fig. 1). The laboratory findings showed a close to normal total bilirubin level $(1.4 \mathrm{mg} / \mathrm{dl})$, high levels of cholestatic markers (alkaline phosphatase $=1236 \mathrm{U} / \mathrm{L}$; gama glutamil transferase $=1282 \mathrm{U} / \mathrm{L}$; total cholesterol $=249 \mathrm{mg} / \mathrm{dl}$ ). Tumor markers - CEA, AFP, CA19-9 - were within normal range. The condition was initially considered as a stage IV Klatskin tumor and was referred for palliative chemotherapy. After a reassessment in our 
tertiary HPB center, the condition was recognized as a PCC of the left hepatic duct (LHD) arising on a anatomical variant with the right posterior hepatic duct (bile duct for segments 6 and 7 according to Couinaud classification) draining into the LHD, and with the right anterior hepatic duct draining directly into the main bile duct (no right hepatic duct) (fig. 1 and 2). Contrastenhanced abdominal and thoracic computed tomography showed no vascular invasion and no distant metastases. The patient was assessed by our oncology multidisciplinary board that confirmed the diagnosis, and was therefore scheduled for surgery.

The approach was through a Mercedes incision. In order to improve the access to the hepatocaval confluence, the xiphoid process was routinely removed. The intraoperative assessment confirmed the preoperative findings. Intraoperative ultrasound showed invasion of segment 1 . The type of resection was classified according to the anatomical nomenclature of Brisbane 2000 terminology of liver anatomy and resections (8). Consequently, a left hemihepatectomy with en-bloc segment 1 resection, and with hilar lymph node dissection was performed (fig. 2 and 3). The resection was performed using the anterior approach and intraoperative ultrasound guidance that assisted in accurately exposing de middle hepatic vein on the cut surface.

The liver transection was carried out using the crash clamping technique (Kellyclasia) (9), combining ligations (4.0 or 2.0 non-absorbable multifilament suture

Figure 2 - Schematic representations of the biliary anatomical variant, tumor localization, and surgical planning for resection. RA - right anterior hepatic duct; RP - right posterior hepatic duct; C - cystic duct.

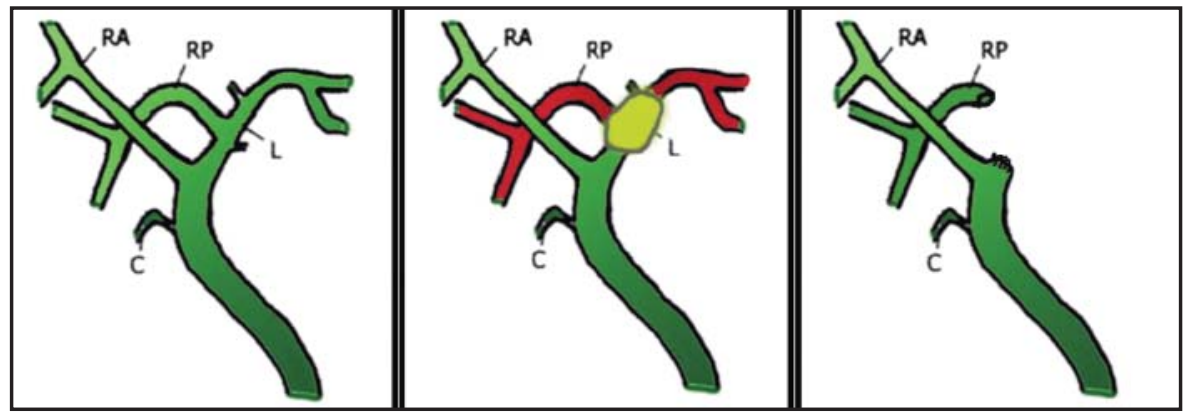

Figure 3 - Intraoperative aspects during left hemihepatectomy with en-bloc segment 1 resection, and with hilar lymph node dissection, preserving the common bile duct and the right anterior hepatic duct. Middle hepatic vein was exposed on the cut surface. Portal vein, hepatic artery and common bile duct may be seen. Inferior vena cava was completely exposed; right hepatic vein was also exposed after resection of the paracaval portion of segment 1 . The stump of the right posterior hepatic duct is indicated by the surgical instrument. Biliary reconstruction was performed for the right posterior bile duct stump, through a Roux-en-Y cholangiojejunostomy.
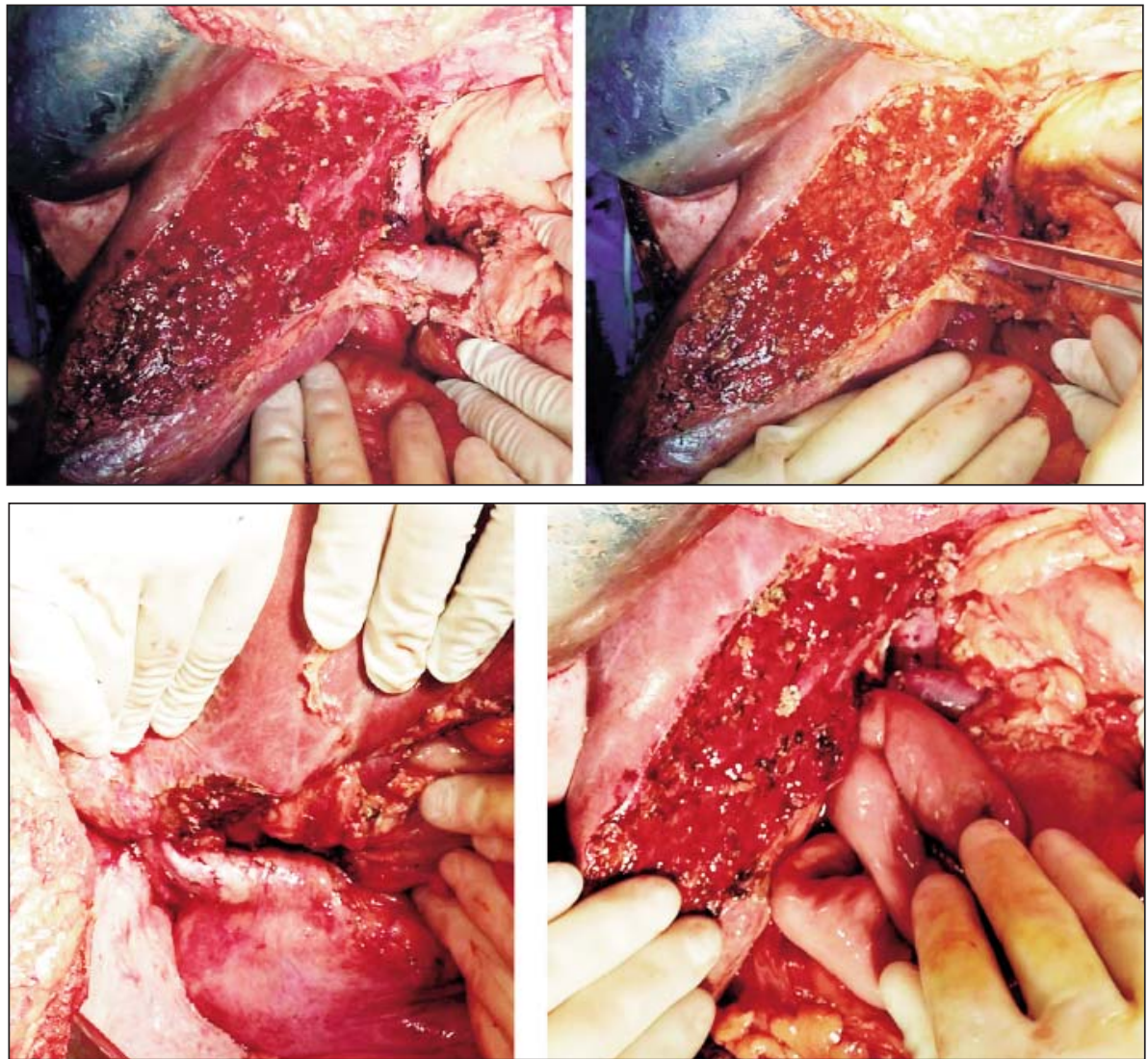
material, depending on the width of the pedicle to be ligated), sutures (4.0 to 6.0 non-absorbable monofilament suture material for vessels, 4.0 to 6.0 absorbable monofilament suture material for bile ducts, and 3.0 or 4.0 absorbable multifilament suture material for parenchyma), and use of monopolar cautery. To limit the backflow bleeding from the hepatic veins during liver transection, the central venous pressure was maintained whenever possible bellow $5 \mathrm{mmHg}$. For vascular control, on demand intermittent portal pedicle clamping (periods of maximum 15 minutes of inflow occlusion with interposed minimum 5 minutes intervals of liver reperfusion) was routinely used in order to limit the ischemiareperfusion injury. The intraoperative blood loss was approximately $750 \mathrm{ml}$; one unit of blood was transfused. The biliary reconstruction was needed only for the right posterior bile duct stump, for which a Rouxen-Y cholangiojejunostomy was performed. The right anterior bile duct was preserved along with the main bile duct. The operative time was $450 \mathrm{~min}$.

The pathological examination of the operative specimen (fig. 4) showed a well to moderated cholangiocarcinoma (G1-G2) of the left bile duct, infiltrating the segment 1 . No invasion of distal part of the resected LHD was encountered (RO resection). Two out of 8 excised hilar lymph nodes were invaded.

The postoperative outcome was remarkably uneventful, and the patient was discharged on the postoperative day 8. Adjuvant chemotherapy with gemcitabine was carried out. The patient died at 23 months postoperatively, due to peritoneal carcinomatosis. It is worth mentioning that no local recurrence was encountered.

\section{DISCUSSION}

Preoperative imaging is very important to establish the diagnosis of PCC, to identify the level of biliary obstruction, and the tumor staging (size, lymph node and vascular invasion, distant metastases). The imaging protocol includes Doppler ultrasound, endoscopic and intraductal ultrasound, contrast-enhanced computed tomography, contrast-enhanced magnetic resonance angiography and cholangiography, endoscopic retrograde cholangiography, percutaneous transhepatic cholangiography, and even positron emission tomography - computer tomography $(10,11,12)$.

Variations in the anatomy of the intrahepatic bile ducts are very common, with serious implications during major hepatectomy for various liver conditions, and also for graft harvesting in living donor liver transplantation. Thus, accurate knowledge of bile duct anatomy and its variants is of utmost importance in such cases. These biliary anatomical variants may be accurately detected at magnetic resonance cholangiography, or endoscopic retrograde cholangiography (13) (14). Among these variants, drainage of the right posterior bile duct into the left hepatic duct is encountered in about $11 \%$ of cases, anomaly defined by Choi et al as type 3A (7).

Our case presented such a biliary variant, with a PCC
Figure 4 - The resected specimen comprising the left hemiliver and the segment 1 . Sectioned tumor shows infiltration of segment 1.

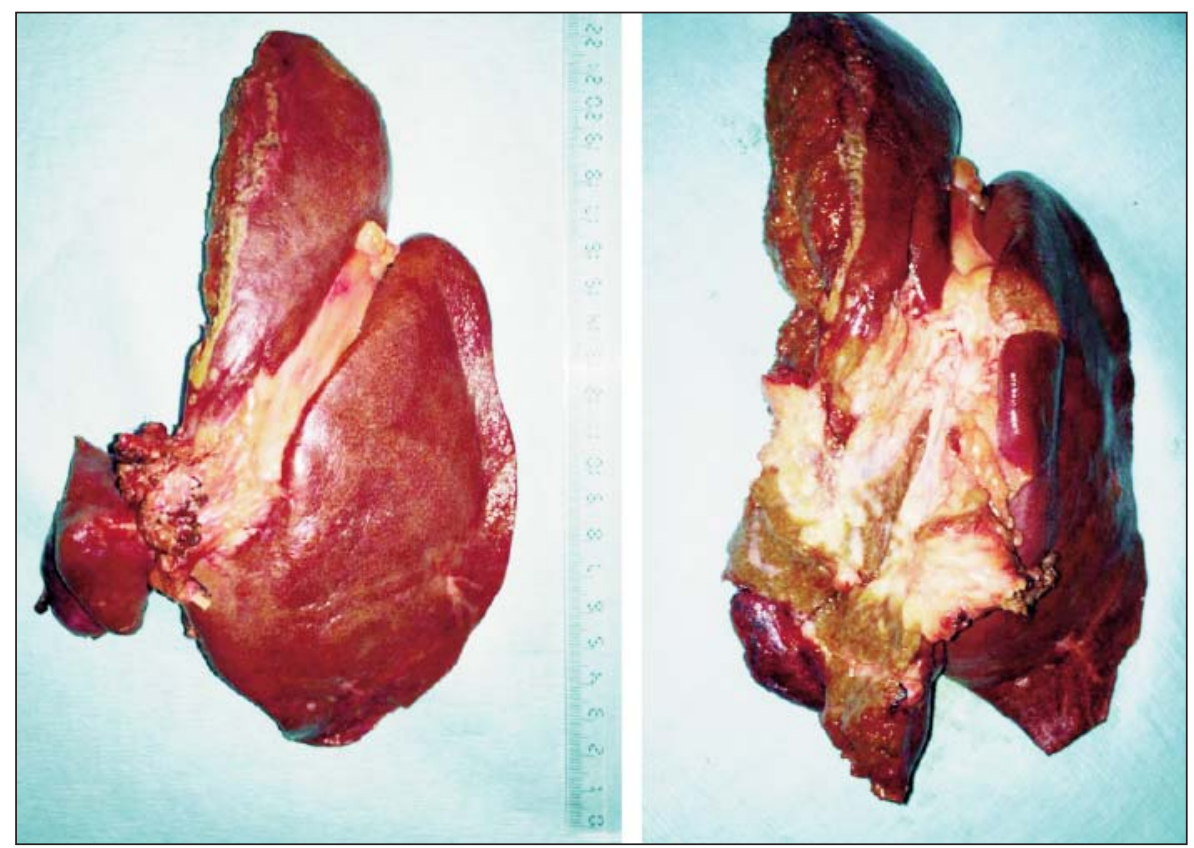


arising from the place where the right posterior hepatic duct entered the left hepatic duct, with consequent bile duct dilatations of the left hemiliver, the right posterior section, and segment 1 , while the bile ducts for the right anterior section were normal. This particular presentation was initially misinterpreted as type IV diffuse Klatskin tumor, and considered unresectable. After a thorough re-evaluation in our tertiary HBP center, the particular real condition was recognized and the patient was referred to surgery.

In PCC, RO resection and hilar lymphadenectomy is the only curative treatment available to date $(15,16$, except liver transplantation in highly selected patients 17). In most cases, the PCC extends to the caudate lobe, so radical caudate lobectomy is recommended for curative treatment, reducing the local recurrence and improving the 5 -year survival $(18,19)$. Complete resection of segment 1 is technically feasible, that may pe performed without significant intraoperative adverse events (20).

Our case benefitted from a left hemihepatectomy with en-bloc segment 1 resection, with hilar lymph node dissection. The resection of segment 1 was performed for oncological safety, as in case of biliary anatomical variants, the paracaval portion of segment 1 also may drain into the left hepatic duct. This was later confirmed at pathology. Furthermore, the resection of segment 1 allowed a wider resection of the right posterior hepatic duct, facilitating, in the same time, the access for performing the cholangiojejunostomy. Segment 1 resection insured the RO resection in this case, as showed at pathology. The number of retrieved lymph nodes $(\mathrm{N}=8)$ was considered optimal, since 7 is the cut-off for proper lymph node count for prognostic staging reported in the literature for PCC (21).

Major hepatic resection for PCC has a morbidity rate of $14-76 \%$, and a mortality rate of $0-15 \%(22,23,24$, 25). Our case had an uneventful postoperative course, and was discharged in POD 8.

PCC has a poor prognosis, with a 5-year survival rate after resection of around $30 \%$ at best, in patients without lymph node invasion. The 5-year survival rate drops to $15 \%$ in patients with regional lymph node metastases, and to $12 \%$ in those with para-aortic lymph node metastases (26). Our patient died 2 years after surgery, with no local recurrence, due to peritoneal carcinomatosis.

\section{CONCLUSION}

Careful preoperative assessment and identification of anatomical variants are often the key to a successful surgical treatment in PCC. En-bloc resection of segment 1 is a safe and useful option for such a patient, in order to achieve negative resection margins.

\section{Conflicts of interest}

The authors declare that there are no conflicts of interest.

\section{REFERENCES}

1. de Groen PC, Gores GJ, LaRusso NF, Gunderson LL, Nagorney DM. Biliary tract cancers. N Engl J Med. 1999;341(18):1368-78.

2. Khan SA, Toledano MB, Taylor-Robinson SD. Epidemiology, risk factors, and pathogenesis of cholangiocarcinoma. HPB (Oxford). 2008:10(2):77-82

3. DeOliveira ML, Cunningham SC, Cameron JL, Kamangar F, Winter $\mathrm{JM}$, Lillemoe KD, et al. Cholangiocarcinoma: thirty-one-year experience with 564 patients at a single institution. Ann Surg. 2007; 245(5):755-62.

4. Chamberlain RS, Blumgart LH. Hilar cholangiocarcinoma: a review and commentary. Ann Surg Oncol. 2000;7(1):55-66.

5. Ragab A, Lopez-Soler RI, Oto A, Testa G. Correlation between 3DMRCP and intra-operative findings in right liver donors. Hepatobiliary Surg Nutr. 2013;2(1):7-13.

6. Nakamura T, Tanaka K, Kiuchi T, Kasahara M, Oike F, Ueda M, et al. Anatomical variations and surgical strategies in right lobe living donor liver transplantation: lessons from 120 cases. Transplantation. 2002;73(12):1896-903.

7. Choi JW, Kim TK, Kim KW, Kim AY, Kim PN, Ha HK, et al. Anatomic variation in intrahepatic bile ducts: an analysis of intraoperative cholangiograms in 300 consecutive donors for living donor liver transplantation. Korean J Radiol. 2003;4(2):85-90.

8. Terminology Committee of the IHPBA. Terminology of liver anatomy and resections. HPB Surg, 2000;2:333-9.

9. Lin TY. A simplified technique for hepatic resection: The crush method. Ann Surg. 1974;180(3):285-90.

10. Sugiura T, Nishio H, Nagino M, Senda Y, Ebata T, Yokoyama Y, et al. Value of multidetector-row computed tomography in diagnosis of portal vein invasion by perihilar cholangiocarcinoma. World J Surg. 2008;32(7):1478-84.

11. Chak A, Catanzaro A. Innovative methods of biliary tract diagnosis: intra-ductal ultrasound and tissue acquisition. Gastrointest Endosc Clin N Am. 2003;13(4):609-22.

12. Sun $L$, Wu H, Guan YS. Positron emission tomography/computer tomography: challenge to conventional imaging modalities in evaluating primary and metastatic liver malignancies. World $\mathrm{J}$ Gastroenterol. 2007;13(20):2775-83.

13. Mortele KJ, Ros PR. Anatomic variants of the biliary tree: MR cholangiographic findings and clinical applications. AJR Am J Roentgenol 2001;177:389-394

14. Gulliver DJ, Cotton PB, Baillie J. Anatomic variants and artifacts in ERCP interpretation. AJR Am J Roentgenol. 1991;156(5): 975-80.

15. Launois B, Terblanche J, Lakehal M, Catheline JM, Bardaxoglou E, Landen S, et al: Proximal bile duct cancer: high resectability rate and 5-year survival. Ann Surg. 1999;230(2):266-75.

16. Madariaga JR, Iwatsuki S, Todo S, Lee RG, Irish W, Starzl TE. Liver resection for hilar and peripheral cholangiocarcinomas: a study of 62 cases. Ann Surg. 1998;227(1):70-9.

17. Rea DJ, Heimbach JK, Rosen CB, Haddock MG, Alberts SR, Kremers WK, et al. Liver transplantation with neoadjuvant chemoradiation is more effective than resection for hilar cholangiocarcinoma. Ann Surg. 2005;242(3):451-8; discussion 458-61.

18. Popescu I, Dumitrascu T. Curative-intent surgery for hilar cholangiocarcinoma: prognostic factors for clinical decision making. Langenbecks Arch Surg. 2014;399(6):693-705. 
19. Rea DJ, Munoz-Juarez M, Farnell MB, Donohue JH, Que FG, Crownhart B, et al. Major hepatic resection for hilar cholangiocarcinoma: analysis of 46 patients. Arch Surg. 2004;139(5):514-23; discussion 523-5.

20. Popescu I, Ciurea S, Romanescu D, Boros M. Isolated resection of the caudate lobe: indications, technique and results. Hepatogastroenterology. 2008;55(84):831-5.

21. Kambakamba $P$, Linecker M, Slankamenac K, DeOliveira ML. Lymph node dissection in resectable perihilar cholangiocarcinoma: a systematic review. Am J Surg. 2015;210(4):694-701.

22. Jarnagin WR, Fong Y, DeMatteo RP, Gonen M, Burke EC, Bodniewicz BS J, et al. Staging, resectability, and outcome in 225 patients with hilar cholangiocarcinoma. Ann Surg. 2001;234(4):507-17; discussion 517-9.

23. Kawasaki S, Imamura H, Kobayashi A, Noike T, Miwa S, Miyagawa S. Results of surgical resection for patients with hilar bile duct cancer: application of extended hepatectomy after biliary drainage and hemihepatic portal vein embolization. Ann Surg. 2003; 238(1):84-92.

24. Baton D, Azoulay D, Adam DV, Castaing D. Major hepatectomy for hilar cholangiocarcinoma type 3 and 4: prognostic factors and longterm outcomes. J Am Coll Surg. 2007;204(2):250-60. Epub 2006 Dec 27

25. Nagino M, Ebata T, Yokoyama Y, Igami T, Sugawara G, Takahashi Y, et al. Evolution of surgical treatment for perihilar cholangiocarcinoma: a single-center 34-year review of 574 consecutive resections. Ann Surg. 2013;258(1):129-40.

26. Kitagawa Y, Nagino M, Kamiya J, Uesaka K, Sano T, Yamamoto H, et al. Lymph node metastasis from hilar cholangiocarcinoma: audit of 110 patients who underwent regional and para-aortic node dissection. Ann Surg. 2001;233(3):385-92. 